\title{
PET/CT Versus CT In Post-Operative Follow Up Of Breast Cancer Patients
}

\author{
Hala H. Mohamed ${ }^{1}$, Osama M. Ebied ${ }^{1}$, Mohamed A. Hussein ${ }^{2,}$ * \\ ${ }^{1}$ Department of Radiodiagnosis, Faculty of Medicine, Menoufia University, Menoufia, Egypt \\ ${ }^{2}$ Department of Radiodiagnosis, Shareq El-Madina Hospital, Alexandria, Egypt
}

Email address:

moh_abdel_azeem@yahoo.com (M. A. Hussein)

\section{To cite this article:}

Hala H. Mohamed, Osama M. Ebied, Mohamed A. Hussein. PET/CT Versus CT In Post-Operative Follow Up Of Breast Cancer Patients. International Journal of Medical Imaging. Vol. 3, No. 2, 2015, pp. 28-33. doi: 10.11648/j.ijmi.20150302.14

\begin{abstract}
Objectives: The aim of this work is to compare the sensitivity and specificity of CT alone versus PET/CT in postoperative follow up of breast cancer patients. Background: Positron emission tomography (PET), using 18Ffluorodeoxyglucose (FDG) is based on the principle of increased glucose metabolism in malignant tumors and has been frequently investigated in breast cancer. Materials \& Methods: Thirty female patients with breast cancer have been investigated. All patients had positive operative history. PET/CT studies were performed for post-operative follow up. The inclusion criteria in this study are histologically proved breast cancer, positive operative history and normal blood urea and creatinine levels. Exclusion criteria is pregnancy and renal impairment. Patients were divided into three groups, first group FDG PET/CT was requested for non-conclusive CT findings, second group patients referred for FDG PET/CT for elevated tumor markers, while in third group FDG PET/CT was requested for follow up after chemotherapy and/or radiotherapy. Results: A comparison of the sensitivity and specificity for CT alone versus PET/CT in each group, in the first group CT alone had sensitivity and specificity of $90 \%$ and $12.5 \%$ respectively, while PET/CT had sensitivity and specificity $100 \%$. In the second group CT alone had sensitivity and specificity of $57 \%$ and $100 \%$ respectively, while PET/CT had sensitivity and specificity of $100 \%$. In group three, CT alone had sensitivity and specificity of $83.3 \%$ and $84.25 \%$, respectively and PET/CT had sensitivity of $83.3 \%$ and specificity of $100 \%$.Conclusions: PET/CT is more sensitive and specific than CT alone for post-operative follow up of breast cancer patients.
\end{abstract}

Keywords: Positron Emission Tomography, Computed Tomography, Breast

\section{Introduction}

Breast cancer is the most common cause of cancer death among women worldwide. In Egypt, breast cancer is the most common cancer among women which representing about $17.5 \%$ of total cancer cases received at the Egyptian National Cancer Institute from 2003 to 2004. [1]

The accurate staging of local, regional, and distant recurrences after initial diagnosis and treatment is critical for therapeutic planning. In general, systemic therapy is used at almost all disease stages; however, isolated local-regional disease or a single site of metastatic recurrence is also treated with surgery and radiation therapy. After treatment, follow-up examinations are required for the early detection and accurate staging of recurrences. [2]

FDG PET has high accuracy for the diagnosis of recurrent or metastatic breast cancer; FDG PET provides functional information, and it often complements conventional imaging modalities, which are more dependent on morphologic changes to depict disease recurrence. FDG PET is particularly useful for discriminating between viable tumor and post-therapy changes such as necrosis or fibrotic scarring in patients with equivocal results of anatomic imaging. FDG PET is also useful in patients whose only indicator of cancer recurrence is an increase in the serum levels of tumor markers such as carcinoembryonic antigen or CA 15-3 antigen. [3]

Clinicians usually refer patients for PET/CT scan when conventional imaging studies are equivocal, suggestive or considered as high risk according to patient's histological and surgical manifestations. [4]

FDG PET/CT may be useful for evaluating asymptomatic treated breast cancer patients with rising levels of tumor markers without clinical symptoms. In this clinical scenario, FDG PET/CT allows more accurate diagnosis of metastatic 
disease compared with conventional imaging. [5]

\section{Patients and Methods}

This study included thirty Patients after approval from the ethical committee, and informed consents were obtained from them. Those patients were referred to the Radiological diagnosis \& nuclear medicine departments at Sharq Elmadina Hospital, Alexandria, Egypt for post-operative follow up by $\mathrm{PET} / \mathrm{CT}$.

Full history was taken from every patient including patient operative, histopathological and lab data.

Patients included in this study were subdivided into three sub-groups; Patients with non-conclusive CT findings represent (Group-1). This group of patients showed nonconclusive CT findings and PET-CT was requested to estimate the metabolic activities of the $\mathrm{CT}$ positive lesions, as well as to confirm the negativity of the CT scans.

Group-2 patients; which had elevated tumor markers and PET-CT was requested to detect any new developed lesions responsible for this elevation.

Group-3 patients had positive history of chemotherapy and/or radiotherapy; PET-CT was requested for follow up.

The procedure was explained to the patients and they were asked to fast for six hours prior to the scan. All metallic items were removed. In addition patients were asked to empty the bladder. In diabetic patients; serum glucose was routinely measured prior to $18 \mathrm{~F}-\mathrm{FDG}$ injection, and fasting levels were 80-210 ng/dl.

An I.V. cannula was inserted in the patient's arm for administration of $18 \mathrm{~F}-\mathrm{FDG}$. The patients were instructed to avoid any kind of strenuous activity prior to the examination and following injection of the radioisotope to avoid physiologic muscle uptake of FDG.

One liter of negative oral contrast agent (5\% mannitol) was given approximately one hour before the study and IV injection of of 3-7 MBq/kg of $18 \mathrm{~F}-\mathrm{FDG}$ was given within time 45-90 minutes before examination. This period is referred to as the uptake phase and is the necessary amount of time for the FDG to be adequately bio-distributed and transported into the patient's cells. Patients were asked to rest in a quiet room, devoid of distractions, and they were also asked to keep their movements, including talking, at an absolute minimum level. This minimizes physiologic uptake of FDG into skeletal muscles, which is important for interpretation of the scan. Patients lie supine on the PET/CT machine with arms above the head.

Helical CT was performed following injection of $125 \mathrm{ml}$ of a low- osmolarity iodinated contrast medium at a rate of 4 $\mathrm{ml} / \mathrm{sec}$ using a power injector. For a typical whole body PET/CT study (skull, neck, chest, abdomen, and pelvis), scanning begins at the level of the skull and extends caudally to the level of the upper thighs. The total length of CT coverage was an integral number of bed positions scanned during acquisition of PET data. The study was performed with the patient breathing quietly. Scanning parameters were collimation width of $5.0 \mathrm{~mm}$, pitch of 1.5 , gantry rotation time of 0.8 second and field of view of $50 \mathrm{~cm}$. The helical data were retrospectively reconstructed at one $\mathrm{mm}$ intervals.

PET was performed following the CT study without moving the patient. Approximately six to seven bed positions were planned for the three dimensional acquisition modes for scanning the entire patient with 3-5 minute acquisition at each bed position.

Trans-axial PET and CT images were first reconstructed, and then reformatted into coronal and sagittal images to facilitate image interpretation. For each of these sets of PET and CT images, corresponding fusion images, combining the two types of data, were also generated. The whole acquisition time for an integrated PET/ CT scan was approximately 2530 minutes. PET image data sets were reconstructed using CT data for attenuation correction and co-registered images were displayed using special software.

Standardized uptake value (SUV) was measured for suspicious lesions; malignant lesions have an SUV greater than 2.5-3.0, whereas normal tissues such as the liver, lung, and marrow have SUVs ranging from 0.5 to 2.5

\section{Results}

Among Group-1 patients (see Table-1); 17 lesions were detected by CT, 10 of them are positive in PET/CT and 7 are negative. One of the negative lesions is seen in the operative bed, 2 axillary lymph nodes and 4 bone metastasis, as well as one osteoblastic metastasis is seen in combined PET/CT and not seen in the conventional CT.
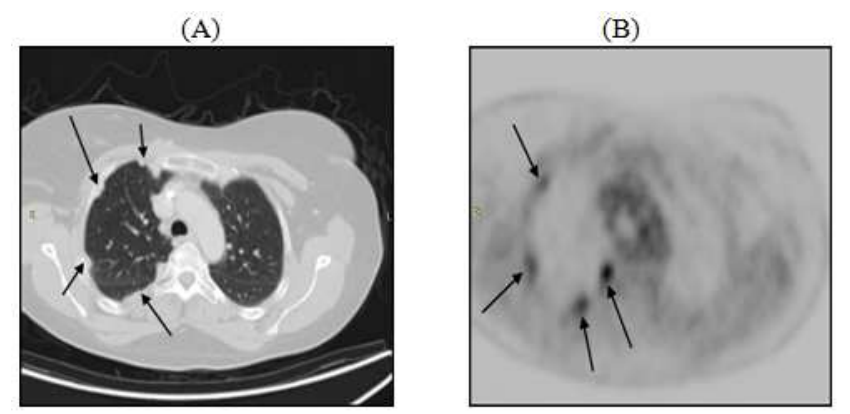

(C)

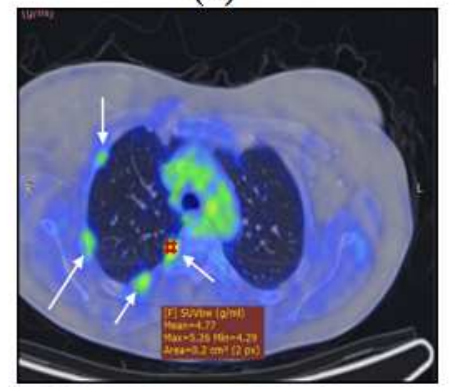

Group 1. patient; a 51 years old female with pathologically proven right ductal carcinoma underwent right lumpectomy with axillary dissection, presented with CT-based nodular pleural thickening. (A) Axial CECT - Lung window: multiple pleural nodules of the anterior, posterior and the lateral pleural surfaces of the right lung (arrows). (B) Axial PET: showing abnormal area of increased FDG uptake (arrows). (C) Axial Fusion PET/CT: revealing SUVmax of about 5 in the forementioned nodules. 
(A)

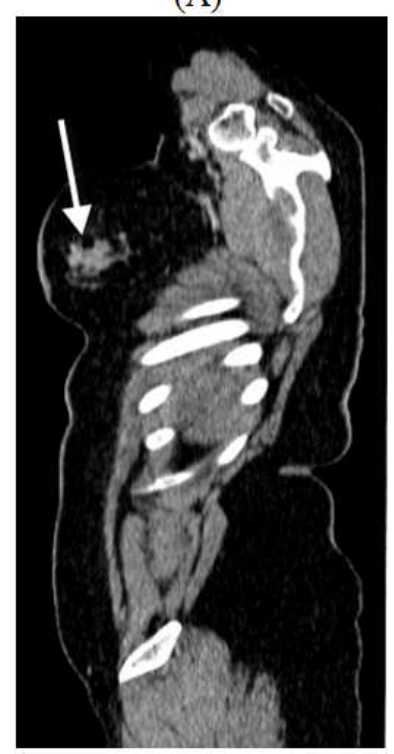

(B)

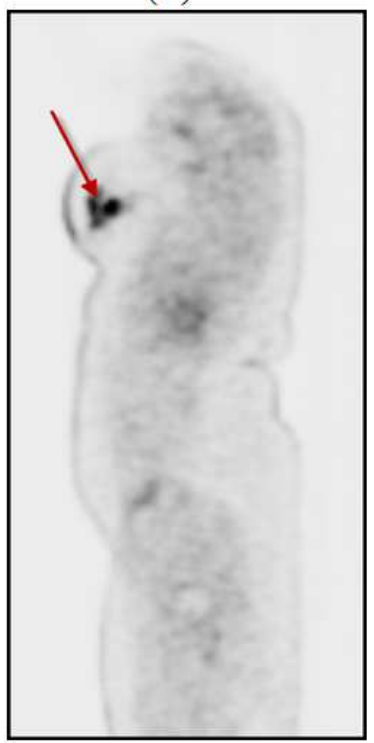

(C)

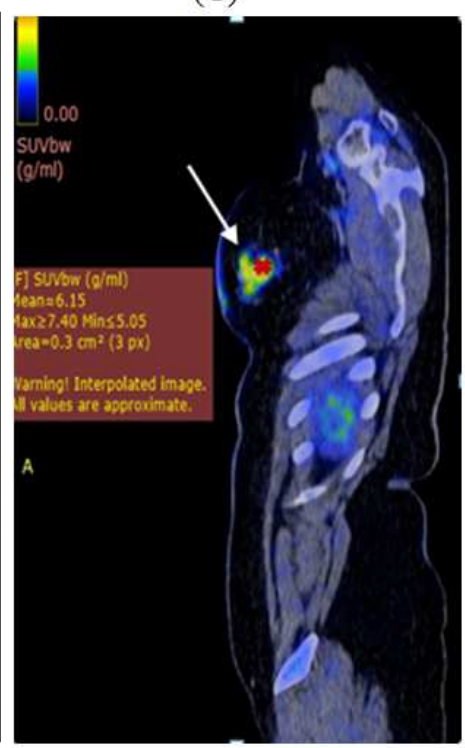

Group 2. patient; a 47 years female with pathologically proven right lobular carcinoma underwent right lumpectomy with axillary dissection, presented with elevated tumor markers. (A) Sagittal CECT: an ill-defined right breast mass measuring about $5.2 X 4.2 \mathrm{~cm}$ with stranding of the surrounding fat planes (arrow). (B) Sagittal PET: showing ill-defined area of increased FDG uptake related to the right breast (arrow). (C) Sagittal Fusion PET/CT: revealing SUVmax of about 7.4 in the aforementioned breast lesion(arrow).

Among Group-2 patients (see Table-2); 8 lesions were detected by CT, all of them are positive in PET/CT. 6 patients are known to be false negative (CT-based); one seen in the operative bed, 2 axillary lymph nodes, 1 bone metastasis, and 2 visceral metastasis. Both $\mathrm{CT}$ and combined PET/CT has zero false positive results for this group.

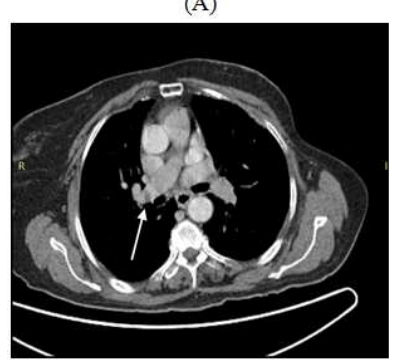

(C)

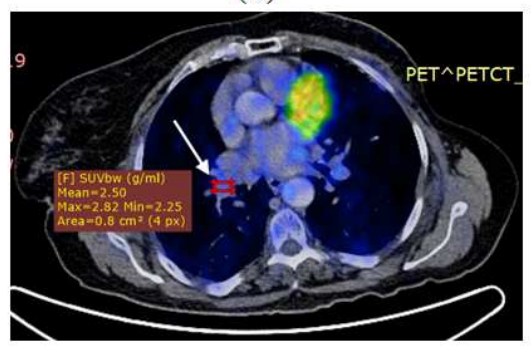

Group 3. patient; a 69 years female with pathologically proven left ductal carcinoma with left modified radical mastectomy \& axillary dissection, presented for follow up after CTH.(A) Axial CECT: ill-defined enlarged right hilar lymph node measuring about $1.2 \times 8 \mathrm{~cm}$ showing central breakdown (arrow). (B) Axial PET: showing no abnormal metabolic activity. (C) Axial Fusion PET/CT: revealing SUVmax of about 2.5 in the aforementioned $L N$, denoting no significant activity
Among Group-3 patients (see Table-3); 9 lesions were detected by $\mathrm{CT}, 5$ of them are positive and all of the five are detected in PET/CT, 3 are known to be false positive in malignant $\mathrm{LN}$ and 2 visceral metastatic lesions, and not seen in combined PET/CT. One false negative (bone metastasis lesions) is not seen in either PET/CT or CT but seen in bone scan.

\section{Discussion}

Positron emission tomography is a functional imaging modality that is increasingly used worldwide. F-18 FDG PET imaging is widely used in clinical oncology. Broadly, the use of FDG PET in breast cancer imaging can be discussed in terms of evaluation of the primary lesion, evaluation for distant metastatic disease, and evaluation of treatment response. PET has also been proposed as a useful modality for monitoring the response of breast cancer patients to chemotherapy, and as guiding choice of therapy. [6]

$\mathrm{PET} / \mathrm{CT}$ imaging of the breast offers physiological information complementary to that achieved from conventional imaging techniques and, therefore, can be used as better characterization of the disease. [7]

Assessment of the response to therapy by PET/CT is based on reduction of the tumor FDG uptake, which represents reduced glucose metabolism in the cells which reflects cell death. Preliminary studies on small cohorts of patients have shown a correlation between early reduction of tumor FDG uptake and clinical response after completion of neo-adjuvant chemotherapy. [8]

The patients included in this study were subdivided into three groups according to the referring cause for PET/CT post-operative follow up. 
The first group consisted of twelve patients who were referred because of non-conclusive findings in the follow up $\mathrm{CT}$. The results showed only one false negative lesion and the calculated sensitivity of CT alone for this group was $90 \%$. The added functional imaging of PET in combined PET/CT raised it to $100 \%$. CT alone also showed seven false positive lesions with a specificity of $12.5 \%$, but PET-CT showed $100 \%$ (see Table-1).

This agrees with the study by Jung et al, [9] on the clinical significance of FDG-PET/CT in the post-operative surveillance of breast cancer patients. The calculated PET/CT sensitivity was $97.5 \%$ and the overall specificity was $98.8 \%$.

Pennant $\mathrm{M}$ et al. (10) compared the combined PET/CT versus conventional imaging modalities including $\mathrm{CT}$ alone, PET and MRI for the diagnosis of breast cancer recurrence. In that study, combined PET/CT had significantly high values of sensitivity and specificity (up to $95 \%, 97 \%$ respectively), compared with conventional imaging modalities including CT. Combined PET/CT proved to have a clear advantage over CT alone for the diagnosis of breast cancer recurrence and had great impact on patients' therapy.

Veit-Haibach P. [11] studied the role of FDG-PET/CT in restaging of patients with recurrent breast cancer, and compared the accuracy of combined PET/CT versus CT alone in restaging patients in the follow up studies. Overall TNM tumor restaging was correctly determined in 40/44 patients with $\mathrm{PET} / \mathrm{CT}$ and in 36/44 patients with $\mathrm{CT}$ alone. Combined PET/CT appeared to be more accurate in reassessing the TNM staging and showed better impact on therapy over PET or CT alone.

For post-operative follow up of breast cancer patients, tumor markers play an important role in early detection for relapsing cases, thus permitting rational choice of therapy. Despite this role of tumor markers as gatekeepers for further exploration, several patients with negative marker profiles have been demonstrated to have clinical evidence of tumor recurrence or metastasis. [12]

There were nine patients in the second group of this study presenting with elevated tumor markers, CT showed sex false negative lesions and the sensitivity of $\mathrm{CT}$ was calculated to be $57 \%$. Combined PET/CT raised it to reach $100 \%$. On the other hand the specificity of CT and combined PET/CT was 100\%. (see Table-2).

Kurata et al. [13] studied post-operative monitoring of breast cancer patients by PET/CT. It was found to be more sensitive in detection of recurrence than serum tumor marker levels in patients with clinical findings suggestive of recurrent breast cancer. All the patients in their study who had elevated serum tumor marker levels and had negative conventional imaging findings had positive PET/CT findings.

Another study by Champion L et al. [14] of breast cancer recurrence diagnosis suspected on tumor marker rising and the value of whole-body 18 FDG-PET/CT imaging and its impact on patient management stated that 18FDG-PET/CT imaging is an efficient technique to detect breast cancer recurrence suspected on tumor marker rising in asymptomatic patients. It may be contributed to improve patient management, providing an earlier diagnosis with complete whole-body staging as an excellent procedure.

Filippi V et al. [15] evaluated the clinical impact of FDGPET-CT scan on restaging breast cancer patients with rising tumor markers while conventional imaging studies had negative or equivocal findings and none of their patients had obvious clinical signs of relapse. On the basis of their results, FDG-PET/CT had a sensitivity of $86.8 \%$, and a specificity of $87.5 \%$. Clinical management was affected in $50 \%$ of their patients and FDG-PET/CT played an important role in overall restaging of their patients.

The third group in this study (9 patients), presented for follow up after treatment by chemotherapy and/or radiotherapy. The sensitivity of $\mathrm{CT}$ alone and of combined PET/CT was the same $83.3 \%$ with only one false negative lesion in each. CT alone showed one false positive lesion with a specificity of $84.21 \%$, while combined PET/CT specificity reached $100 \%$ (see Table-3). The overall relatively low combined PET/CT sensitivity for this group was because neither CT alone nor combined PET/CT could detect osteogenic bone metastasis that was only detected by bone scintigraphy. This agrees with Gallowitsch et al. [16] who found that although PET provided better lesion-based specificity, it exhibited poorer sensitivity for the detection of bone metastases (specificity $88.9 \%$, sensitivity $56.5 \%$ ) than bone scintigraphy (specificity $74.1 \%$, sensitivity $89.8 \%$ ). PET yielded a large proportion of false-negative lesions in cases with bone metastasis, and most of the lesions that were not detected by PET were osteogenic or mixed osteogenic/ osteolytic lesions. These results imply that PET is not a substitute for bone scanning.

The skeleton is the most common site of distant metastasis in breast cancer; nearly $70 \%$ of patients who have advanced disease have bone metastasis. Bone scintigraphy is considered the most sensitive method for detecting and determining the extent of skeletal metastases. However, purely osteolytic lesions or metastases confined to the marrow cavity may be difficult to detect on bone scintigraphy because of lack of sufficient osteoblastic response. [17]

In the study by Nakai et al. [18] 23 breast cancer patients who had known skeletal metastases underwent both bone scintigraphy and FDG-PET. FDG-PET detected more lesions than bone scintigraphy, except in a subgroup of patients who had osteoblastic metastases. Moreover, the level of FDG uptake in osteolytic lesions was significantly greater compared with osteoblastic lesions, and the prognosis of patients who had osteolytic-predominant disease was significantly worse.

Several studies. [19] comparing the sensitivity of bone scintigraphy to FDG/PET, in the detection of skeletal metastases in patients who have advanced disease, have shown conflicting results. Some studies have shown FDGPET to be equal or superior to planar bone scintigraphy in the detection of skeletal metastases whereas others have shown FDG-PET to be less sensitive on a lesion-based analysis.

Finally, the overall sensitivity of CT for the three groups in this study was calculated to be $74.19 \%$, the added functional 
imaging of PET in combined PET/CT raised this percentage to reach $96.88 \%$. The overall specificity of CT alone was $64.29 \%$, and in combined PET/CT reached $100 \%$ (see Table4). This agrees with the study by Port et al. [20] on 80 patients with operable breast cancer. The rate of falsepositive results with conventional imaging (CT or bone scintigraphy) was greater than FDG PET (17\% vs. 5\%). The findings on conventional imaging generated additional tests and biopsies that eventually had negative results.

Greco et al. [21] studied 167 breast cancer patients, and axillary involvement was detected in 68 of 72 patients, resulting in a sensitivity of $94.4 \%$ and a specificity of $86.3 \%$; overall accuracy of lymph node staging with PET was $89.8 \%$.

Our study agrees with Andrei et al [22] study of breast $18 \mathrm{~F}$ FDG PET/CT and MRI in the management of breast cancer, demonstrating that PET/CT with overall sensitivity of $85 \%$ has an important role in detecting distant metastasis of breast cancer. This help to plan surgical and medical treatment, and monitoring response to treatment.

In addition, our results agree with that of Constantinidou $\mathrm{A}$ et al. [23] stating that PET/CT is equally specific and more sensitive than other imaging modalities in detecting small lesions (5-10 $\mathrm{mm})$, particularly lymph nodes as well as visceral and bone disease. A significant proportion of their patients had PET/CT scans for staging in the recurrent and metastatic disease, especially in cases of rising tumor markers. $\mathrm{PET} / \mathrm{CT}$ provided accurate assessment of the metastatic disease in all these cases and revealed more metastatic sites than those identified by CT. It was more accurate than bone scintigraphy in detecting metastatic osteolytic disease. One of the other important findings in their study is that PET/CT is useful in accurate assessment of response to chemotherapy and hormonal therapy. They stated that there is a strong relationship between the response to therapy and the decrease in FDG signal even at an early stage of therapy.

\section{Conclusion}

FDG-PET-CT is generally more sensitive in the detection of distant metastases than conventional imaging, and is therefore a more accurate method of determining the recurrence of the disease. One exception is the detection of sclerotic bone metastases; these lesions are often not metabolically active enough for FDG-PET detection, and bone scintigraphy could help in such cases.

FDG-PET-CT seems to be highly useful for monitoring response to therapeutic interventions. This technique can identify response to therapy earlier than any other imaging method currently available which greatly improves patient management by allowing termination of ineffective and toxic therapies.

PET-CT proved to be helpful in the evaluation of anatomic regions that have been previously treated by surgery or radiation in which the discrimination between post-treatment scar and recurrent tumor can be problematic

Table (1). Sensitivity and specificity table for group-1 patients.

\begin{tabular}{lllllllll}
\hline & \multicolumn{7}{c}{ CT } & \multicolumn{7}{c}{ PET/CT } \\
\cline { 2 - 8 } & True positive & True negative & False positive & False negative & True positive & True negative & False positive & False negative \\
\cline { 2 - 8 } & 10 & 1 & 7 & 1 & 12 & 7 & - & - \\
Sensitivity & $90 \%$ & & & & $100 \%$ & & \\
Specificity & $12.5 \%$ & & & & $100 \%$ & & & \\
\hline
\end{tabular}

Table (2). Sensitivity and specificity table for group-2 patients.

\begin{tabular}{lllllllll}
\hline & CT & & \multicolumn{2}{c}{ PET/CT } \\
\cline { 2 - 8 } & True positive & True negative & False positive & False negative & True positive & True negative & False positive & False negative \\
\cline { 2 - 7 } & 8 & 1 & - & 6 & 14 & 1 & - & - \\
Sensitivity & $57 \%$ & & & & $100 \%$ & & \\
Specificity & $100 \%$ & & & $100 \%$ & & & \\
\hline
\end{tabular}

Table (3). Sensitivity and specificity table for group-3 patients.

\begin{tabular}{|c|c|c|c|c|c|c|c|c|}
\hline & \multicolumn{4}{|l|}{ CT } & \multicolumn{4}{|l|}{ PET/CT } \\
\hline & True positive & True negative & False positive & False negative & True positive & True negative & False positive & False negative \\
\hline & 5 & 16 & 3 & 1 & 5 & 19 & - & 1 \\
\hline Sensitivity & $83.3 \%$ & & & & $83.3 \%$ & & & \\
\hline Specificity & $84.21 \%$ & & & & $100 \%$ & & & \\
\hline
\end{tabular}

Table (4). Overall Sensitivity and specificity for CT and PET/CT for all groups.

\begin{tabular}{|c|c|c|c|c|c|c|c|c|}
\hline & \multicolumn{4}{|l|}{$\mathrm{CT}$} & \multicolumn{4}{|l|}{ PET/CT } \\
\hline & True positive & True negative & False positive & False negative & True positive & True negative & False positive & False negative \\
\hline & 23 & 18 & 10 & 8 & 31 & 27 & - & 1 \\
\hline Sensitivity & $74.19 \%$ & & & & $96.88 \%$ & & & \\
\hline Specificity & $64.29 \%$ & & & & $100 \%$ & & & \\
\hline
\end{tabular}




\section{References}

[1] Hala S El Rebey, Hayam A Aiad, Nancy Y Asaad, Moshira M Abd El-Wahed, Iman L Abulkheir, Fatma M Abulkasem, et al. Immunohistochemical expression of topoisomerase II a and tissue inhibitor of metalloproteinases 1 in locally advanced breast carcinoma, Menoufia Medical Journal 2014; 27 : 1.

[2] Gallowitsch HJ, Kresnik E, Gasser J, Kumnnig G, Igerc I, Mikosch P, et al. F-18 fluorodeoxyglucose positron-emission tomography in the diagnosis of tumor recurrence and metastases in the follow-up of patients with breast carcinoma: a comparison to conventional imaging. Acta Radiol 2003; 38(5):250.

[3] Murakami R, Kumita S, Yoshida T, Ishihara K, Kiriyama T, Hakozaki $\mathrm{K}$ et al. FDG-PET/CT in the diagnosis of recurrent breast cancer. Acta Radiol 2011; 53(1):12.

[4] Segaert I, Mottaghy F, Ceyssens S, De Wever W, Stroobants S, Van Ongeval $\mathrm{C}$ et al. Additional value of PET-CT in staging breast cancer. Experimental and therapeutic medicine 2010;16:617.

[5] Hodgson NC, GulenchynKY. Is there a role for positron emission tomography in breast cancer staging? Clinical oncology 2008; $26: 721$.

[6] Isasi CR, Moadel R, Blaufox M: A meta-analysis of FDG-PET for the evaluation of breast cancer recurrence and metastases. Breast Cancer Res Treat 2005; 90:105.

[7] Buck AK, Herrmann K, Stargardt T, Dechow T, Krause BJ, Schreyögg J. Economic evaluation of PET and PET/CT in oncology. J Nucl Med 2010; 51:401.

[8] Radan L, Ben-Haim S, Bar-Shalom R, Guralnik L, Israel O. The role of FDG-PET/CT in suspected recurrence of breast cancer. Cancer 2006; 107(11):2545.

[9] Na Young Jung,Ie Ryung Yoo,Bong Joo Kang,Sung Hun Kim,Byung Joo Chae, Ye Young Seo. Clinical significance of FDG-PET/CT at the postoperative surveillance in the breast cancer patients, Radiographics. 2014; 24:1411.

[10] Pennant M, Takwoingi Y, Pennant L, Davenport C, Fry-Smith A, Eisinga A, et al. A systematic review of positron emission tomography (PET) and positron emission tomography/computed tomography (PET/CT) for the diagnosis of breast cancer recurrence. Health Technol Assess 2010; $14: 1$.

[11] Veit-Haibach P, Antoch G, Beyer T, Stergar H, Schleucher R, Hauth EA. FDG-PET/CT in restaging of patients with recurrent breast cancer: possible impact on staging and therapy. Br J Radiol 2007; 80(955):508.

[12] Cheung K, Graves CRL, Robertson JFR. Tumor marker measurements in the diagnosis and monitoring of breast cancer. Cancer Treat Rev 2000; 26:91.
[13] Kurata A, Murata Y, Kubota K, Osanai T, Shibuya H. Multiple 18F-FDG PET-CT for postoperative monitoring of breast cancer patients, Acta Radiol 2009; 50: 979, DOI: $10.3109 / 02841850903147046$

[14] Champion L, Brain E, Giraudet AL, Le Stanc E, Wartski M, Edeline V, et al. Breast cancer recurrence diagnosis suspected on tumor marker rising: value of whole-body $18 \mathrm{FDG}-\mathrm{PET} / \mathrm{CT}$ imaging and impact on patient management. Cancer 2011; 117:1621

[15] Filippi V, Malamitsi J, Vlachou F, Laspas F, Georgiou E, Prassopoulos $\mathrm{V}$ et al. The impact of FDG-PET/CT on the management of breast cancer patients with elevated tumor markers and negative or equivocal conventional imaging modalities. Nucl Med Commun, 2011; 32(2):85.

[16] Gallowitsch HJ, Kresnik E, Gasser J, Kumnig G, Igerc I, Mikosch P et al. [F-18]-fluorodeoxyglucose positron-emission tomography in the diagnosis of tumor recurrence and metastases in the follow-up of patients with breast carcinoma: a comparison to conventional imaging. Invest Radiol; 2003; $38(5): 250$.

[17] Cook G, Houston S, Rubens R, M N Maisey, I Fogelman. Detection of bone metastases in breast cancer by 18FDG PET: differing metabolic activity in osteoblastic and osteolytic lesions, Journal of Clinical Oncology. 1998; 16, (10):3375.

[18] Nakai T, Okuyama C, Kubota T, Yamada K, Ushijima Y, Taniike $\mathrm{K}$ et al. Pitfalls of FDG-PET for the diagnosis of osteoblastic bone metastases in patients with breast cancer. Eur.J.Nuc.Med.Mol.Imaging 2005; 32:1253.

[19] Yang SN, Liang JA, Lin FJ, Kao CH, Lin CC, Lee CC. comparing whole body [F-18]-2-deoxyglucose positron emission tomography and [technetium-99m]-methylene diphosphonate bone scan to detect bone metastases in patients with breast cancer. J Cancer Res Clin Oncol; 2002; 128(6):325.

[20] Port ER, Yeung H, Gonen M, Liberman L, Caravelli J, Borgen $\mathrm{P}$, et al. 18F-2-fluoro-2-deoxy-D-glucose positron emission tomography scanning affects surgical management in selected patients with high-risk, operable breast carcinoma. Ann Surg Oncol. 2006; (5):677.

[21] Greco M, Crippa F, Agresti R. Axillary lymph node staging in breast cancer by 2-Fluoro-2- deoxy-D-glucose-positron emission tomography: Clinical evaluation and alternative management. J Natl Cancer Inst. 2001; 93(8):630.

[22] Andrei I, Rinat M, Sravanthi K, Peter S. Breast MRI and 18F FDG PET/CT in the management of breast cancer. Annals of Nuclear Medicine. 2007;21;(1):33.

[23] Constantinidou A, Martin A, Sharma B, Johnston SR. Positron emission tomography/computed tomography in the management of recurrent/metastatic breast cancer: a large retrospective study from the Royal Marsden Hospital. Ann.Oncol.2011; 22:307. 